\title{
Propylthiouracil embryofetopathy
}

INSERM

\section{Source}

INSERM. (1999). Orphanet: an online rare disease and orphan drug data base.

Propylthiouracil embryofetopathy. ORPHA:485358

Propylthiouracil embryofetopathy is a rare teratologic disease characterized by variable congenital anomalies resulting from maternal treatment and prenatal exposure to propylthiouracil. Anomalies frequently encountered include ear malformations (e.g. accessory auricle, preauricular sinus/fistula/cyst), urinary system malformations (e.g. isolated unilateral kidney, congenital hydronephrosis), gastrointestinal anomalies (e.g. congenital bands with intestinal malrotation) and cardiac defects (e.g. situs inversus dextrocardia, cardiac outflow tract defects). 